\title{
Akuter Harnverhalt
}

\author{
VON P. КокоTт
}

Ein multimorbider, chronisch kranker 70-jähriger Patient (u. a. Diabetes mellitus, KHK) kann plötzlich kein Wasser mehr lassen. Er wartet zunächst ab, sucht dann aber wegen zunehmender, unerträglich werdender Schmerzen eine Krankenhausambulanz auf. Bekannt ist bei ihm eine Prostatahyperplasie (Prostatavolumen $120 \mathrm{ml}$ ), die vom Urologen kontrolliert wird. Seine Medikamente (Tamsulosin o,4/Spasmolyt ${ }^{\circledR} 10 \mathrm{mg}$ ) hat der Patient regelmäßig eingenommen.

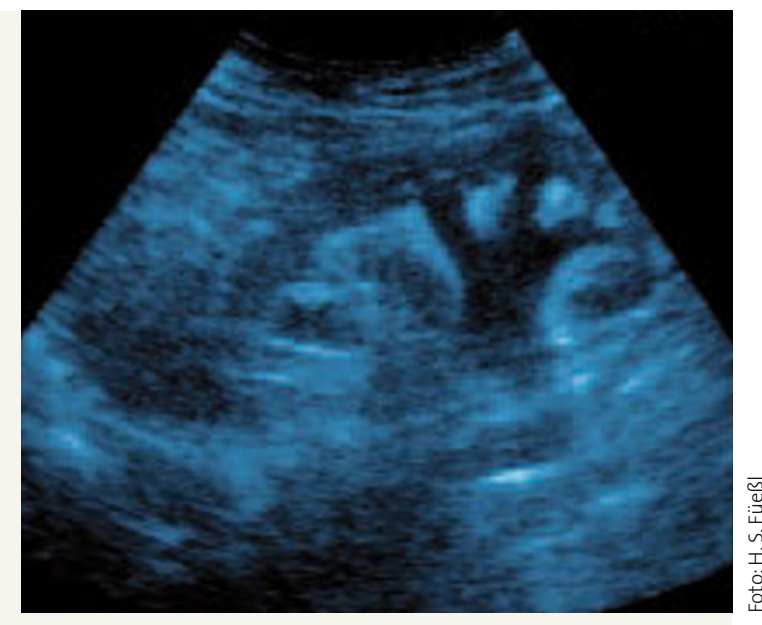

Harnstau mit erweitertem Nierenbecken.

\section{Wie ging es weiter?}

Im Krankenhaus werden unter der Diagnose eines Harnverhalts mit konsekutiver Harnstauungsniere beidseits bei Prostatahyperplasie durch Legen eines transurethralen Blasenkatheters die Beschwerden behoben. Später erfolgt eine transurethrale Resektion der Prostata, wobei sich histopathologisch die Diagnose einer myoglandulären Prostatahyperplasie ohne Malignitätsnachweis bestätigt. Seitdem ist der Patient in dieser Hinsicht beschwerdefrei und kann wieder spontan und restharnfrei miktionieren.

\section{Definition}

Die Harnverhaltung (Harnsperre) wird als völliges Unvermögen, die volle Harnblase zu entleeren, definiert. Bei Überdehnung der Blasenwand bis zur Grenze des Fassungsvermögens kommt es zur Überlaufblase mit Harntröpfeln.

\section{Ätiologie}

Häufigste Ursachen einer Harnverhaltung sind Abflusshindernisse im Bereich der unteren Harnwege und neurogene Blasenentleerungsstörungen (z. B. diabetische autonome Neuropathie). Es lassen sich vesikale und infravesikale Harnabflusshindernisse unterscheiden:

- Harnblasentumor, Harnblasensteine, Harnblasentamponade - Prostataadenom, Prostatakarzinom. Die häufigste Ursache des Harnverhalts beim Mann ist die gutartige Vergrößerung der Prostata (benigne Prostatahyperplasie/BPH), ein Krankheitsbild, das über 50\% der Männer über 60 Jahren betrifft. - Harnröhrenstriktur, Harnröhrenklappe, Harnröhrenpolyp, Fremdkörper

- Harnröhrenabriss (Unterbauchtrauma)

- Neurogene und psychogene Ursachen (z. B. DetrusorSphinkter-Dyssynergien, Diskusprolaps)

- Medikamente (Anticholinergika, Antidepressiva, Neuroleptika).

Davon abzugrenzen sind mögliche Ursachen einer supravesikalen Obstruktion der Harnwege mit nachfolgender Anurie (Harnsteine, Harnleitertumoren, -strikturen, Nierenbeckenab- gangsenge, Ureterkompression, iatrogene Ureterligatur). Eine seltene Form der postrenalen Anurie ist die Reflexanurie.

\section{Klinische Symptomatik}

Die akute Verlegung der ableitenden Harnwege führt zu einer schmerzhaften Miktionssperre oder bei einer inkompletten Okklusion zur Pollakisurie, Dysurie, Dranginkontinenz oder auch zur Algurie. In der Regel ist ein suprapubischer prallelastischer Tumor sichtbar oder palpabel.

Besteht eine Blasenentleerungsstörung über einen längeren Zeitraum, kommt es sekundär zu Veränderungen der oberen Harnwege mit Harnstauung. Dies bedingt mit der Zeit eine Verschlechterung der Nierenfunktion bis hin zur Urämie. Bei bewusstseinsgestörten Patienten kann eine zunehmende motorische Unruhe auf die Miktionssperre hinweisen.

\section{Diagnostik}

Basisdiagnostik: Anamnese, körperliche Untersuchung (Palpation des Unterleibs, rektale Untersuchung), Sonografie.

Weiterführende Diagnostik: Zystoskopie, Zystogramm, Miktionszystogramm, Ausscheidungsurogramm (AUG).

\section{Therapie}

Nach Diagnosestellung sollte eine Drainage der Blase durch sterilen transurethralen Katheterismus erfolgen. Katheter der Wahl ist ein 16-Charrière-Katheter mit Tiemannspitze. Die Harnröhre sollte durch Instillation eines Gleitmittels (z. B. Instillagel oder Cathejell) gleitfähig gemacht werden.

Gelingt die Katheterisierung nicht (übergroße Prostata, Prostatakarzinom, Harnröhrenstriktur), ist eine suprapubische Harnblasenpunktion in Betracht zu ziehen (lange 1erKanüle).

Ziel der weiteren Therapie ist die möglichst rasche Wiederherstellung eines ungestörten Harnabflusses.

\section{Anschrift des Verfassers:}

Dr. med. Paul Kokott, Facharzt für Allgemeinmedizin,

Stormstr. 21, D-38226 Salzgitter 